\title{
Numerical Optimization of a SCR System Based on the Injection of Pure Gaseous Ammonia for the NOx Reduction in Light-Duty Diesel Engines
}

\author{
Augusto Della Torre, Gianluca Montenegro, Angelo Onorati, Tarcisio Cerri, Enrico Tronconi, \\ and Isabella Nova Politecnico di Milano
}

Citation: Della Torre, A., Montenegro, G., Onorati, A., Cerri, T. et al., "Numerical Optimization of a SCR System Based on the Injection of Pure Gaseous Ammonia for the NOx Reduction in Light-Duty Diesel Engines," SAE Technical Paper 2020-01-0356, 2020, doi:10.4271/2020-01-0356

\section{Abstract}

S elective Catalytic Reduction (SCR) systems are nowadays widely applied for the reduction of $\mathrm{NOx}$ emitted from Diesel engines. The typical process is based on the injection of aqueous urea in the exhaust gases before the SCR catalyst, which determines the production of the ammonia needed for the catalytic reduction of NOx. However, this technology is affected by two main limitations: a) the evaporation of the urea water solution (UWS) requires a sufficiently high temperature of the exhaust gases and b) the formation of solid deposits during the UWS evaporation is a frequent phenomenon which compromise the correct operation of the system. In this context, to overcome these issues, a technology based on the injection of gaseous ammonia has been recently proposed: in this case, ammonia is stored at the solid state in a cartridge containing a Strontium Chloride salt and it is desorbed by means of electrical heating.

In this work, an after-treatment system based on the injection of gaseous ammonia in the SCR system is considered.
Numerical 1D and 3D CFD simulations are applied in order to optimize the NOx reduction process. In particular, CFD methodology is applied to study in details the process of injection of the gaseous ammonia in the main exhaust gas stream and the effectiveness of the mixing process. Different geometrical layouts are compared to evaluate their performances in terms of uniformity of the $\mathrm{NH} 3$ distribution across the inlet section of the catalyst and pressure drop introduced in the exhaust line. Moreover, a 1D simulation tool is applied to evaluate the performances of the entire exhaust after-treatment system. The 1D model is calibrated on the basis of the information coming from detailed CFD simulations, in particular for what concerns the modeling of the effects of the different mixer geometries in terms on $\mathrm{NH} 3$ distribution. In this case, a simplified 3D catalyst is simulated, to take into account the maldistribution of ammonia at the inlet cross section and to evaluate its impact on the global deNOx performance of the system for different dosing strategies and for different levels of the ammonia maldistribution.

\section{Introduction}

$\mathrm{n}$ the last years, as a response to the increasing environmental concern in many countries worldwide, the legislation has become more and more restrictive on pollutant and greenhouse emissions from the automotive sector [1]. In particular, testing and homologation procedures have been progressively revised, leading to the introduction of the WLTP cycle and the new RDE assessment. The new testing procedures, introduced in Europe with the Euro 6d-temp regulation, aim at making the homologation procedure more representative of the effective conditions experienced by the vehicle on the road, reducing the deviation between lab measurement and real drive emissions. In this context, many factors could contribute to determine the overall emissions from the vehicle: these includes driver attitude, environmental conditions, specific features of the urban track where the test is performed.

In this context the design of the after-treatment system has become a very challenging task, since it requires to act at different levels for the optimization of the system. The design process consists of the optimization of each single component and of their interaction in the system, taking into account the wide range of operating conditions which can occur under real operation. However, nowadays this task can be supported by a large variety of simulation models which have been proposed over the years. These includes $1 \mathrm{D}$ numerical codes, which allow the simulation of the engine system $[\underline{2}, \underline{3}, \underline{4}]$, and CFD simulation models, which are usually applied for the detailed analysis of the single component [5] . The optimization of the after-treatment system is therefore a process which should integrate the information coming from different tools. In particular, $1 \mathrm{D}$ tools could provide the overall prediction of the performances of the system, identifying the critical components which need to be optimized and the parts of the system which are not crucial for the achievement of the target. On the basis of these information, a detailed CFD analysis can be set up for the components which need to be optimized, eventually applying automatic optimization strategy to 
improve their design. At the same time, the detailed CFD analysis could provide the necessary information needed to improve the simplified 1D model of the component, on the basis of more advanced quasi-3D model which need a proper calibration.

In this work, a methodology which integrates $1 \mathrm{D}$ and CFD models to achieve the optimization of the after-treatment system will be described and applied. The study will be focused on a modern light-duty Diesel engine, equipped with a state-of-the-art after-treatment system including electrical heating, Diesel oxidation catalyst, two stages for NOx reduction, Diesel particulate filter, ammonia slip catalyst. Peculiarity of this system is the injection of gaseous ammonia instead of traditional urea water solution [6] to achieve the NOx reduction in the SCR stages. This alternative technology is based on the storage of the ammonia at the solid state in a cartridge containing a Strontium Chloride salt. The desorption and production of gaseous ammonia, to be injected before SCR section, is controlled by electrical heating on the basis of the instantaneous request. Few examples of this technology are already in production for bus and truck applications, while a large scale application on the automotive sector seems to be limited by the size of the ammonia storage and delivery system. However, this technology shows interesting features, since the injection can be performed even at low temperature, not being affected by the problem of the evaporation as for UWS injection. Moreover, it is not affected by the phenomenon of the formation of solid deposits, which is a typical problem which could compromise the correct operation of the system based on the UWS.

The present paper is structured as follow. At first an overview of the methodology applied in this work will be provided, describing the different simulation approaches adopted and how the information is exchanged between the different level of the analysis. Then the case study will be described, highlighting the target of the optimization. Finally simulation results will be analyzed, pointing out the main achievements in terms of component optimization and its influence on the overall system performances.

\section{Methodology}

The optimization of an after-treatment system requires to analyze the problem at different levels. In particular, the process should focus at the first stage on the analysis of each component, in such a way to characterize its physical behavior and identify the main guidelines for its optimization. Then, when a proper characterization is available for each device, a more integrated approach can be applied in order to investigate the performances of the overall system. In this work, the analysis has been carried out considering two different simulation approaches, namely CFD and $1 \mathrm{D}$ model. The first has been adopted for detailed simulations focused on the single device considered as stand-alone, the latter has been applied for the overall study of the entire aftertreatment system.

\section{Detailed CFD Model of the Single Component}

The CFD model adopted for detailed component analysis was based on the open-source finite-volume code OpenFOAM [ $\underline{7}$, 8]. This has been widely extended, in previous works by the authors, by means of the implementation of specific libraries for the thermal and reacting simulation of the catalytic devices (TWC, DOC, SRC) commonly present on the exhaust lines of modern ICEs []. Moreover, the code includes a flexible and automatic mesh generator, named snappyHexMesh, which provides a body fitted grid, predominantly consisting of hexahedral cells, with a small percentage of polyhedral elements near the boundary walls. Hexahedral/prism boundary layers can be added in order to accurately describe near wall gradients. The capability of directly providing a high quality grid starting from the CAD geometrical description of the component makes this tool a good candidate to be embedded in an automatic geometrical optimization procedure, as will be explained in the following section.

\section{Geometrical Optimization Strategy}

In order to optimize the design of the mixer a CFD guided optimization procedure has been implemented. The methodology is based on the integration of different tools as reported in Figure 1. The optimization procedure is controlled by Dakota [9], which is a general purpose optimizer, developed at Sandia National Labs, which implements many optimization algorithms and can be easily integrated with other tools. In this framework, Dakota run an iterative procedure, determining the values of the variables to test on the basis of the responses of the model. At each iteration, a novel geometry is generated by means of FreeCAD, which is a open-source and free CAD modeler which can manage parametrized models and it is able to automatically change the geometry on the

FIGURE 1 Overview of the optimization procedure based on the integration of Dakota, FreeCAD and OpenFOAM.

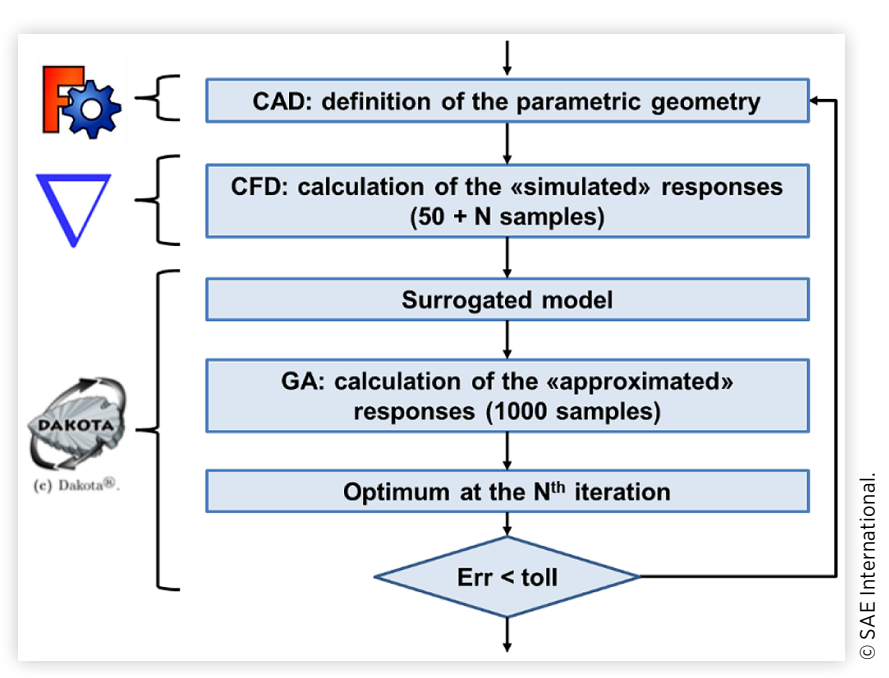


basis of the updated list of geometrical variables. Then, when the updated geometry is available, the determination of the responses of the model is managed by OpenFOAM, which handle the generation of the computational grid, the execution of the fluid-dynamic simulation and the post-processing. On the basis of the actual responses of the CFD model, Dakota creates a surrogate model, which basically consists of an interpolation of the actual responses in order to provide a more detailed approximated response function. The optimization is run on the surrogate model by means of suitable optimization algorithm (e.g.: genetic algorithm). The optimum solution, determined on the surrogate model, is then verified computing the actual response of the model and evaluating the error: if this is higher of the desired tolerance the surrogated model is updated and novel iterations are performed up to convergency. In this work Gaussian process method (Kriging) has been applied to generate the surrogate model, which is at first created on the basis of 50 actual responses of the CFD model.

\section{Overall 1D Modeling of the Entire ATS System}

The modeling of the entire after-treatment system was performed on the basis of the code Axisuite, which implements a quasi-steady state approach to simulate the emission abatement of complex after-treatment systems considering generic test cycle (e.g. NEDC, WLTP, RDE). The code is able to deal with any reaction scheme and rate expressions ("elementary" and "global" reactions) $[\underline{2}, \underline{10}, \underline{3}]$ and includes pre-defined libraries for the modeling of a large set of common catalytic devices. It allows $1 \mathrm{D}, 2 \mathrm{D}$ or $3 \mathrm{D}$ discretization at the device level and 0D, 1D and quasi-2D in the wall direction, used when washcoat diffusion limitations need to be accounted for. Moreover, in order to take into account the non-uniformity of the flow velocity and concentration at the inlet of the monoliths, it could integrate the information coming from detailed CFD modeling of the connecting pipe systems, as it will be shown in a next section.

\section{Case Study}

In this study a standard after-treatment line for a 1.6L Euro 6 Diesel engine was considered. The system consists of four emission abatement stages: a) Diesel Oxidation Catalyst (DOC), where unburned compounds are oxidized; b) Diesel
FIGURE 2 First section of the after-treatment system (eHC, DOC, SDPF) located in close coupled position.

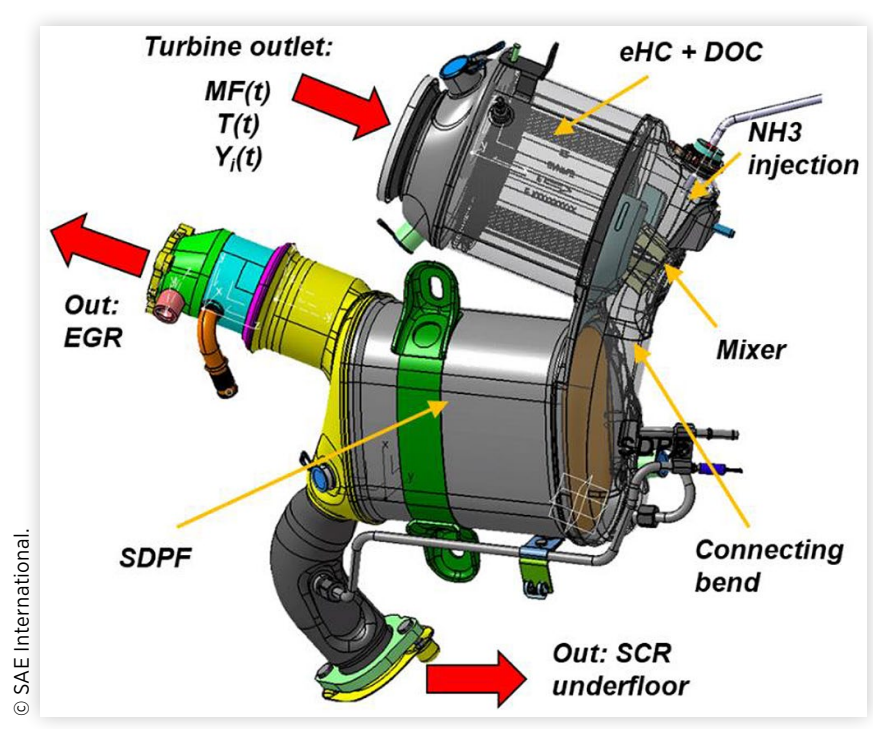

Particulate Filter with SCR coating (SDPF), which is both a filter for particulate matter and the first catalytic stage for nitrogen oxides (NOx) reduction; c) Selective Catalytic Reduction catalyst (SCR), where NOx reduction occurs; d) dual layer Ammonia Slip Catalyst (ASC), where excess ammonia is oxidized in order to prevent ammonia slip to the environment and further NOx reduction takes place. The first section of the system, located in close-couple position, is shown in Figure 6.

Specification for the different catalytic elements inserted on the exhaust line are reported in Table 1. The devices includes commercial catalysts which have been modeled considering standard reaction schemes.

\section{Results and Discussion}

\section{CFD Simulation of the Baseline Configuration}

The detailed CFD analysis was applied to the study the process of injection of the gaseous ammonia in the main exhaust gas stream and the effectiveness of the mixing process. At first the model has been validated considering three different

TABLE 1 Specifications for the different catalytic devices on the exhaust line.

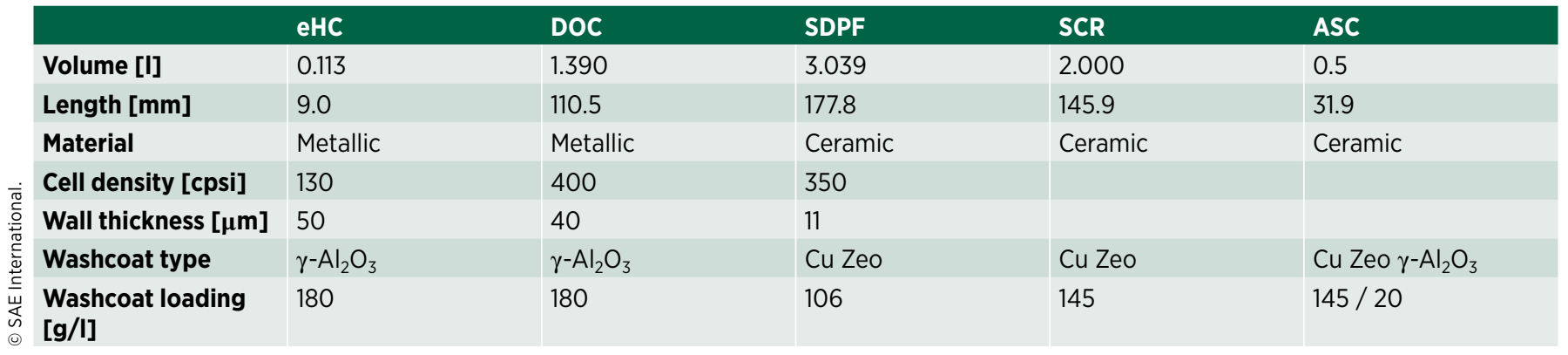


steady-state operating conditions and comparing the results with experimental measurements. Then, the effects of the mixing elements included in the baseline configuration, namely pre-mixer and mixer, have been evaluated, in order to characterize their effects in terms of uniformity of the $\mathrm{NH}_{3}$ distribution at the inlet of the SDPF and pressure drop.

Computational Model The computational grid generated for the simulation of the injection process is reported in Figure 3 . It consists of around $8 \cdot 10^{5}$ cells, with an average cell size of $3 \mathrm{~mm}$. The mesh is refined to $1.5 \mathrm{~mm}$ cell size in the bend connecting DOC and SDPF, where gaseous ammonia is injected and the mixing with the exhaust gas take place. Moreover, additional refinement is applied to capture the geometrical details of specific components, e.g. on the thin walls of the mixer.

Three different steady state operating conditions are simulated, as reported in Table 2 . These are defined by a combination of operating parameters such as: gas flow rate, gas inlet temperature, NOx concentration, ammonia dosing. For these conditions, the experimental characterization of the uniformity of the NH3 distribution at the inlet of the SDPF was available for the validation of the model.

Numerical simulations were run employing a standard SIMPLE algorithm, solving for the transport of the different species involved in the gaseous system [11]. Chemical reactions in the catalyst regions were not taken into account, since the

FIGURE 3 Computational grid for the CFD simulation of the ATS, including DOC and SDPF sections: a) overview and b) detail of the mesh of the internal components in the injection section.

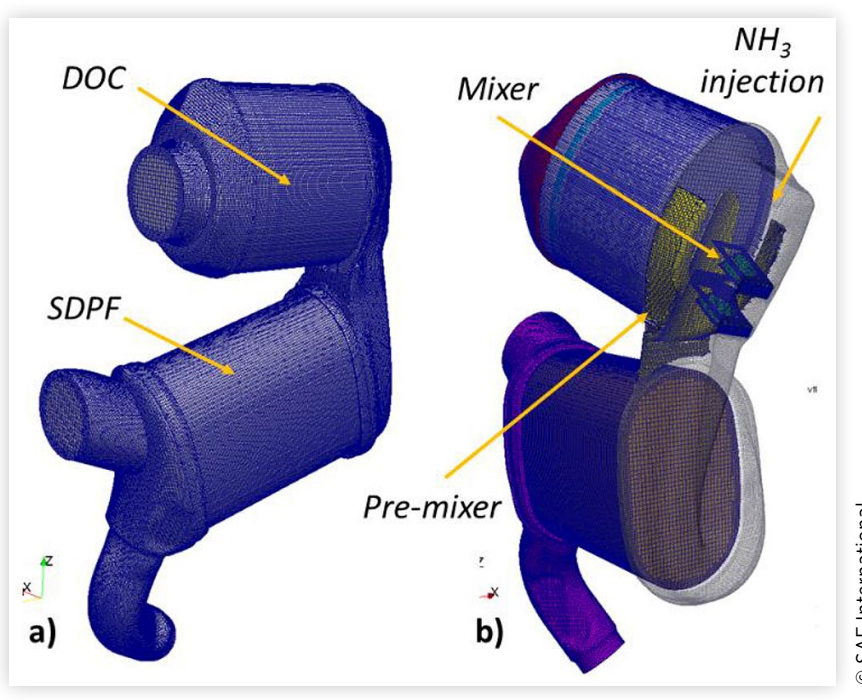

TABLE 2 Steady-state operating conditions simulated

\begin{tabular}{|c|c|c|c|}
\hline & OP1 & OP2 & OP3 \\
\hline Gas flow rate $[\mathrm{kg} / \mathrm{h}]$ & 100 & 300 & 300 \\
\hline Gas temperature, post-DOC $\left[{ }^{\circ} \mathrm{C}\right]$ & 260 & 260 & 410 \\
\hline NOx concentration, post-DOC [ppm] & 200 & 699 & 794 \\
\hline NH3 dosing $[\mathrm{mg} / \mathrm{s}]$ & 2.95 & 30.57 & 58.18 \\
\hline NH3/NOx [-] & 0.9 & 0.9 & 1.5 \\
\hline
\end{tabular}

analysis was purely focused on the description of the mixing process before the SDPF in order to predict the degree of uniformity of the $\mathrm{NH}_{3}$ entering the catalyst. With regards to the turbulence modeling, a standard k- $\omega$ SST model was applied [12]. Moreover, the description of the pressure drop in the catalyst region was managed by means of a non-isotropic Darcy-Forchheimer model, which assumes a quadratic dependency of the pressure drop on the axial flow velocity. On the other hand, the flow permeability is assumed as negligible in the transversal directions. The coefficients of the model are calibrated on the basis of experimental correlations available in the literature for similar catalyst.

Model Validation Computed flow streamlines are reported in Figure 4 for two operating conditions OP1 and OP2, which are characterized by a different mass flow. It can be noticed that the particular shape of the duct connecting the DOC and SDPF, characterized by a spiral design, contributes significantly to distribute quite uniformly the gas flow at the entrance on the SDPF. The gas flow path is similar for the two conditions at low and high mass flow rate, except for some flow recirculation which can be noticed in the bottom part of the bend for OP1.

With regards to the mixing process, it can be seen that the combination of the effect of the pre-mixer plate and the mixer are quite effective in promoting the mixing of the exhaust gas stream and the gaseous ammonia stream injected in the upper part of the connecting duct. Maldistribution on the inlet section of the SDPF was evaluated considering a uniformity index defined as:

$$
U I=1-\frac{\sum\left|X_{i}-\bar{X}\right| A_{i}}{2 \bar{X} \sum A_{i}}
$$

where $X_{i}$ is the local ammonia molar fraction, $\bar{X}$ is the average molar fraction and $A_{i}$ the discretization area. The calculations show that the uniformity index of the $\mathrm{NH}_{3}$ distribution on the inlet section of the SDPF ranges between $83 \%-87 \%$ for all the operating conditions. The visualizations

\section{FIGURE 4 Visualization of the flow streamlines in the} connecting bend for two operating conditions at different mass flow.

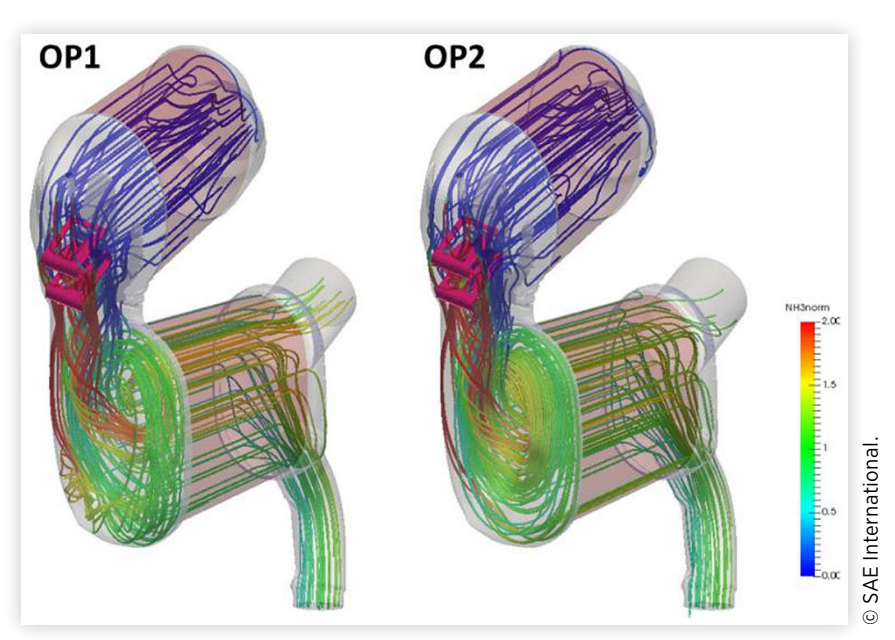


of the ammonia distribution in front of the SDPF, reported in Figure 5 - Figure 7, show the occurrence of two separate zones in terms of ammonia concentration: in particular, $\mathrm{NH}_{3}$ concentration is higher in the upper-left part while it is lower in the bottom-right one. In order to assess the validity of the computational model, results from a preliminary experimental campaign were considered. Ammonia distribution was estimated from the gas concentration measured in 65 points uniformly distributed over the outlet section of the SCR catalyst substrate, considering a test configuration where the downstream ATS components were removed. These experimental findings are in agreement with the simulation predictions, confirming the two-zones distribution of the $\mathrm{NH}_{3}$ on the SDPF inlet section. Moreover, the quantitative comparison of the uniformity index of the ammonia distribution confirms a very good agreement between calculations and experiments for operating conditions OP2 and OP3. On the contrary for

FIGURE 5 Operating condition OP1: comparison between calculated and experimental $\mathrm{NH} 3$ distributions on the inlet section of the SPDF catalyst.

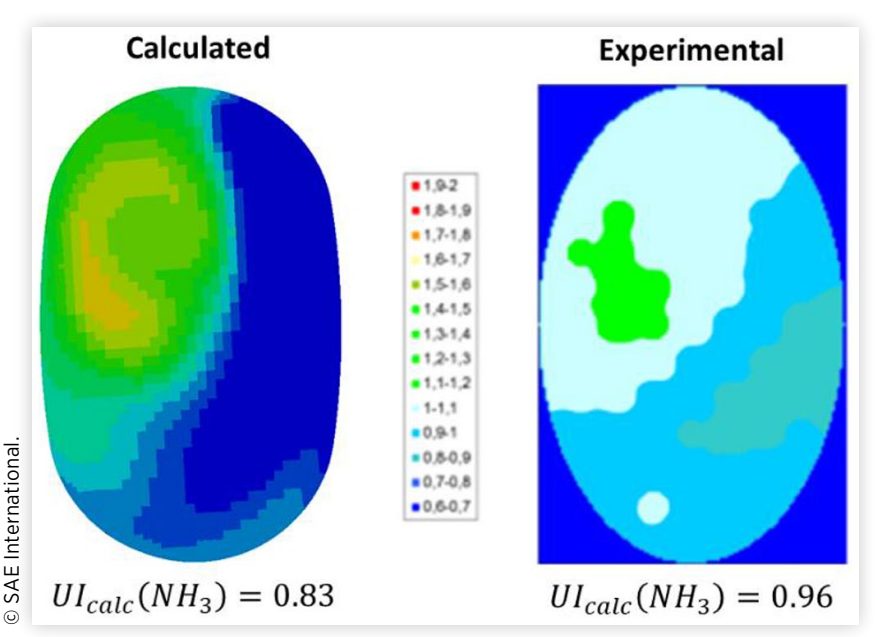

FIGURE 6 Operating condition OP2: comparison between calculated and experimental $\mathrm{NH} 3$ distributions on the inlet section of the SPDF catalyst.

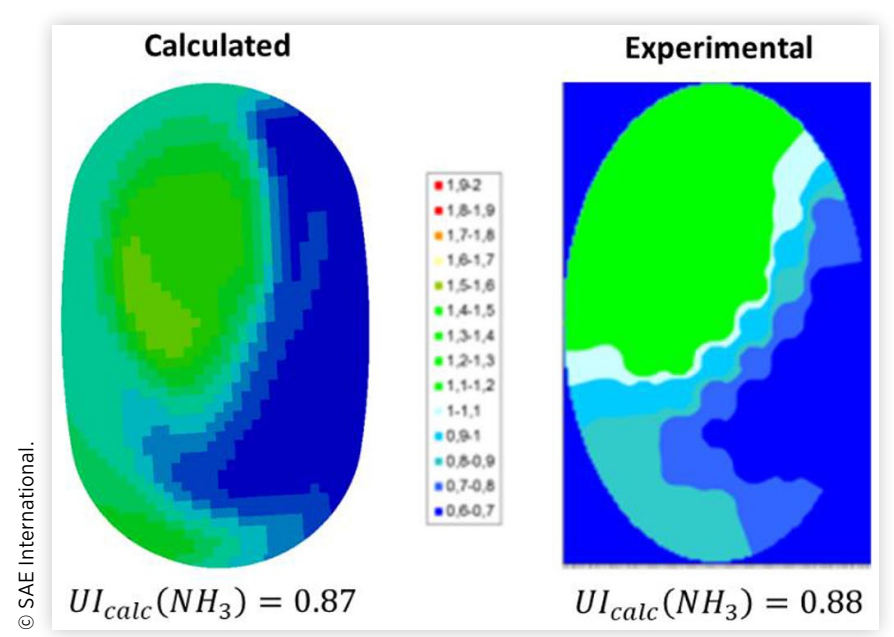

FIGURE 7 Operating condition OP3: comparison between calculated and experimental $\mathrm{NH} 3$ distributions on the inlet section of the SPDF catalyst.

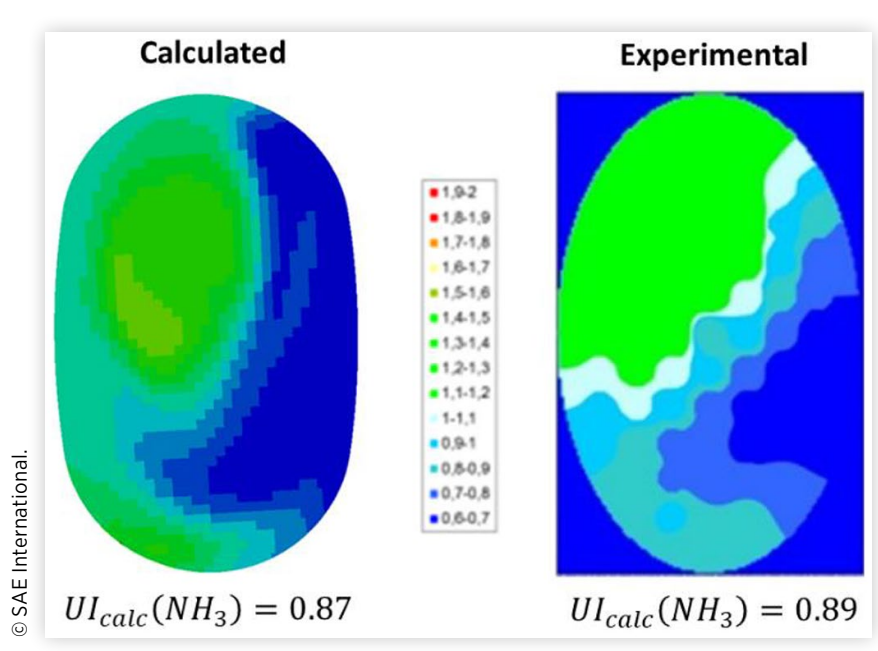

OP1, despite the fact that the overall distribution is qualitatively well captured, the calculated uniformity index significantly underestimates the experimental value. However, further investigations need to be carried out on this particular operating point, since experimental uncertainties may have affected the final measurement, especially in relation of the low mass flow rate (if compared to OP2 and OP3) that has been applied.

Investigation of the Effects of the Mixer/ Pre-Mixer Components As a last step of the preliminary numerical characterization of the baseline configuration, the role of the mixer and pre-mixer components has been investigated. In particular, the analysis focused on the study of the effects of these components in terms of enhancement of the uniformity of the $\mathrm{NH}_{3}$ distribution on the inlet section of the SDPF and in terms of the additional pressure drop introduced on the exhaust line. Two additional configurations, which consist of variations with respect to the baseline, were analyzed and compared:

- variation 1: the configuration in which the mixer is removed, while the pre-mixer plate is still present;

- variation 2: the configuration in which mixer and premixer are both removed, leaving fully opened the gas flow path between the DOC and the SDPF.

Figure 8 shows the effects of the these variations in terms of gas flow streamlines, considering operating conditions OP2. Comparing with the baseline (Figure 4 - OP2), it can be seen that, removing the two mixing components, the vorticity of the flow is significantly reduced as well as the induced mixing between the exhaust and ammonia stream. Moreover, it can be noticed that the presence of the premixer is even disadvantageous in terms of ammonia distribution if not suitably coupled with the effect of the mixer: Figure 9 highlight that, in this condition (variation 1) a significantly high concentration of ammonia is found near the walls in the left part of the catalyst. 
FIGURE 8 Comparison of gas streamlines highlighting the effect of the mixer and pre-mixer components, for operating conditions OP2: var. 1) configuration with only the pre-mixer, without mixer; var. 2) configuration without mixer and premixer.

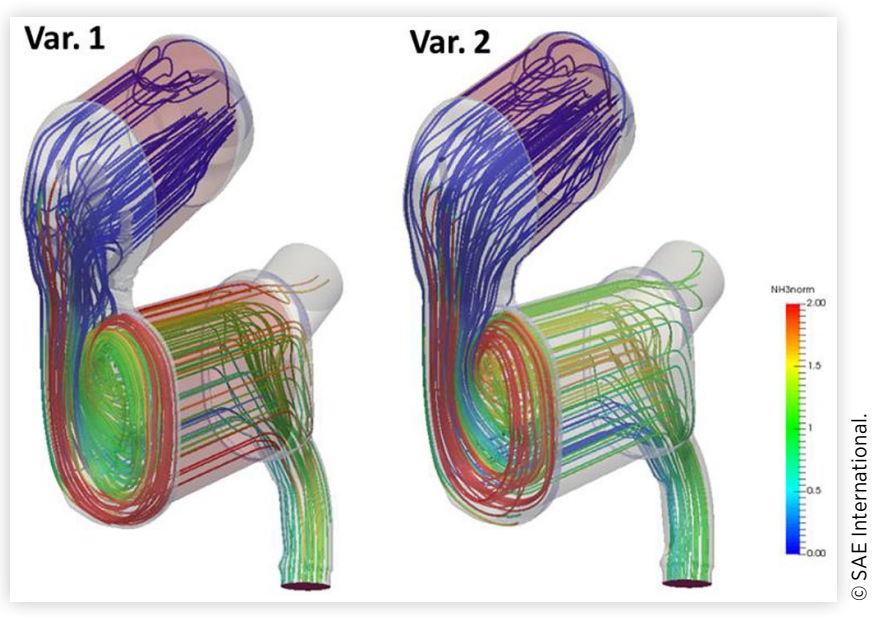

FIGURE 9 Comparison of $\mathrm{NH} 3$ distribution on the SDPF inlet section, for operating conditions OP2: var. 1) configuration with only the pre-mixer, without mixer; var. 2) configuration without mixer and pre-mixer.

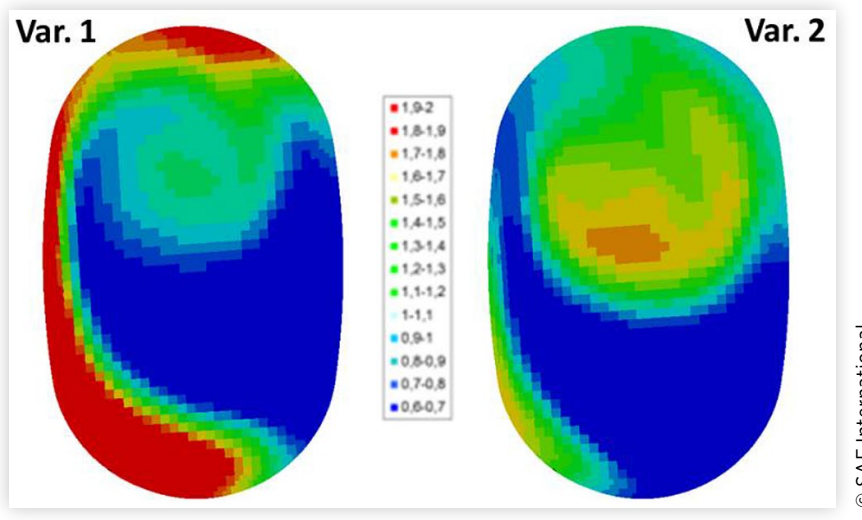

Figure 10-Figure 11 report the detailed results in term of ammonia distribution and pressure drop calculated for the three configuration considering the three operating conditions OP1, OP2, OP3 (as detailed in Table 2). It can be seen that, for all the operating conditions, the baseline configuration exhibits the better performances in terms of uniformity index. On the other hand, the pressure drop introduced by this configuration is significantly higher with respect to the others. Comparing configuration 2 and 3, it can be noticed that the removal of the mixing components determines a progressive and significant reduction of the pressure drop. Instead, for what concern the uniformity index, the effect of the pre-mixer seems to be negligible or even disadvantageous in high flow conditions (OP2 and OP3). This suggests that the mixing function of these two elements is strictly coupled and their design should be conducted considering their interaction in order to optimize the uniformity of the $\mathrm{NH}_{3}$ distribution.
FIGURE 10 Investigation of the effect of mixer and premixer components on the uniformity of the $\mathrm{NH} 3$ distribution on the inlet section of the SDPF catalyst.

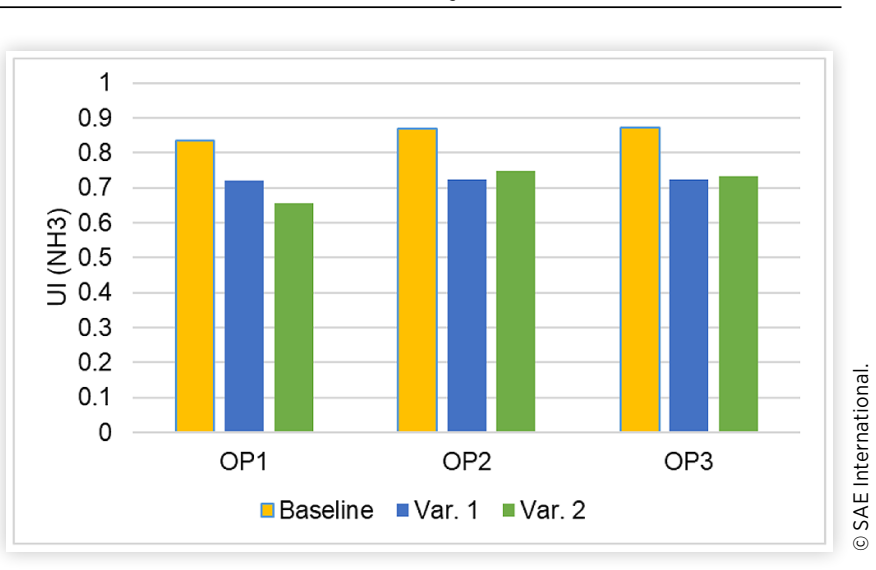

FIGURE 11 Investigation of the effect of mixer and premixer components on the pressure drop related to the mixing section.

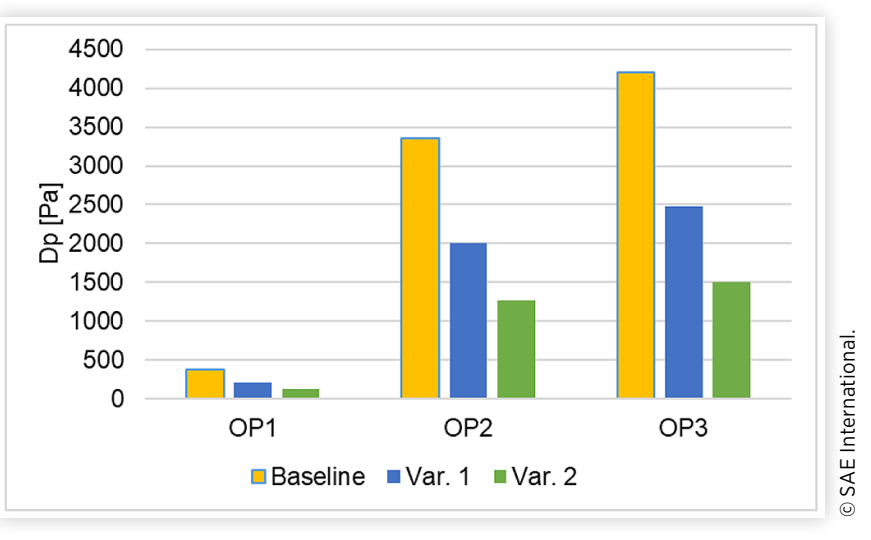

\section{CFD Optimization of an Alternative Mixer Design}

In the previous step the CFD model has been validated and a first characterization of the behavior of the mixing components has been provided, both in terms of effectiveness of the mixing promoted and pressure drop introduced on the exhaust line. On this basis, the second step of the CFD activity focused on the design and the optimization of an alternative configuration characterized by enhanced mixing and reduced pressure drop. In this section the design process of the alternative configuration will be described. At this stage, the optimization will be focused on the stand-alone component, evaluating the performances of the device (mixing and pressure drop) under steady-state representative conditions.

Design of an Alternative Configuration and Overview of Its Main Geometrical Parameters The baseline configuration analyzed in the previous section was designed for the specific case of the injection of a spray of liquid Urea Water Solution (UWS) and it was then adapted for the adoption in a system based on injection of gaseous 
ammonia (ASDS system). Therefore, it results in a quite flexible design which allows to operate the same ATS system alternatively with UWS and ASDS, just changing the injection section and the delivery system. However, looking towards a final configuration in which ASDS will be the preferable system, given its multiple advantages over the UWS (e.g.: superior behavior during low temperature operation, absence of deposits), it becomes interesting to study an alternative mixer specifically designed only for ASDS operation. Its optimization can take advantage by the fact that the mixing process between two gaseous stream is a much easier process to be realized, if compared to the physics of the UWS injection (which includes: droplet formation, evaporation, deposition, stripping, etc). Therefore, the optimum mixer design, which guarantee the desired level of mixing, can be simpler and less penalizing in terms of pressure drop.

The alternative configuration which has been proposed is reported in its base design in Figure 12. It can be seen that the proposed alternative is characterized by a very simple design, consisting in a single perforated plate, curved in the middle and mounted with a certain angle. The mixing is promoted by the deflection of the gaseous streams in the three directions. The holes, which are realized on the plate, have both the function to enhance the mixing and to keep low the pressure drop. Three geometrical parameters (Figure 13) have been identified to be the most significant for the determination

FIGURE 12 Comparison of the baseline mixer configuration and the alternative design which has been proposed.

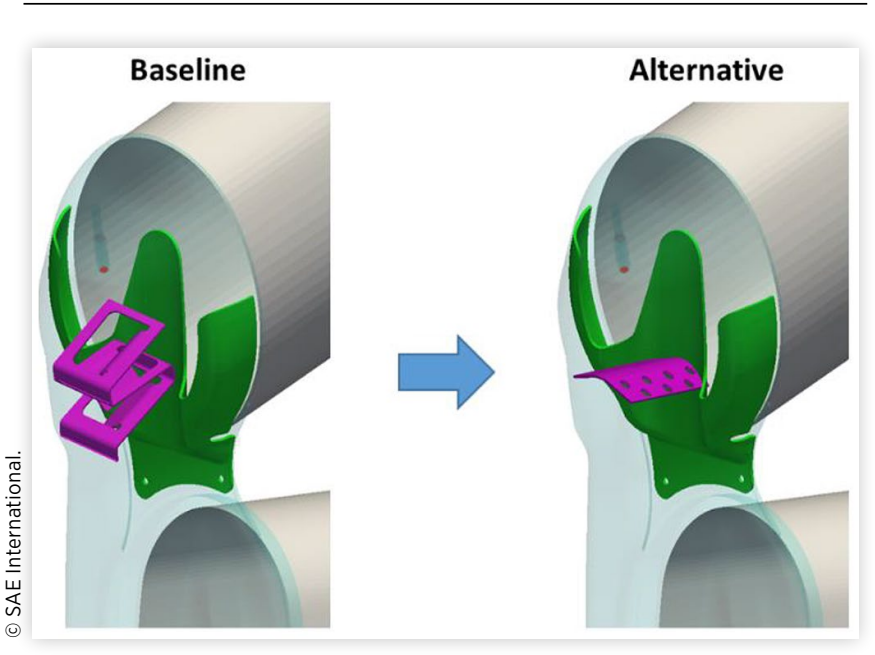

FIGURE 13 Detail of the alternative design: it is parametrized with respect to three geometrical parameters in order to address its geometrical optimization.

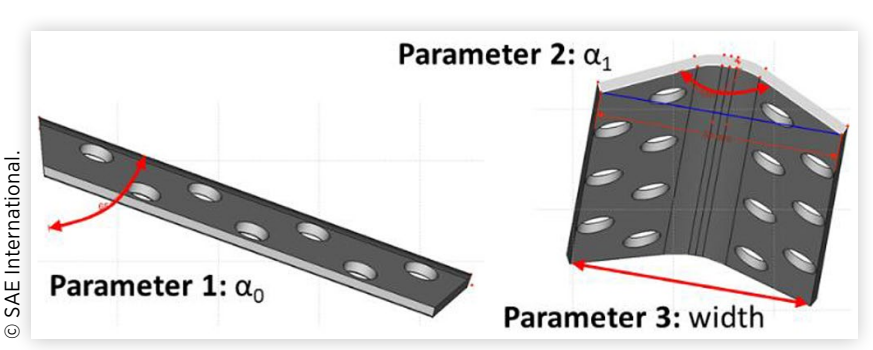

of the behavior of the mixer (i.e.: angle with which the device is mounted, angle determining the lateral deflection of the streams, width of the device) and will be used as variable for the optimization process which will be described in the next sections.

Design Optimization and Final Configuration The optimization strategy described in the previous section "Methodology" was applied to the optimization of the proposed alternative configuration. A Latin Hypercube Sampling (LHS) strategy was selected as method for performing the Design of Experiment (DOE) phase, which is the initial step for the construction of the surrogate model. The combination of geometrical variables chosen by Dakota on the basis of the aforementioned LHS strategy is reported in Figure 14.

In Figure 15-Figure 16 the responses of the model, for each LHS test point, is reported. In particular, two objective functions (uniformity index of the ammonia distribution and pressure drop introduced by the mixing device) are evaluated considering two operating points (operating point OP2 and OP3, as detailed in Table 1). Therefore, the total number of objective functions considered in the optimization is four, while the optimization variables are three. The DOE phase allowed already to find out which are the best configuration, characterized by high uniformity index and low pressure drop: this correspond to iteration \#10, which has been highlighted

FIGURE 14 Combination of values of the geometrical variables for each iteration, chosen by means of LHS and used for the construction of the first surrogate model.

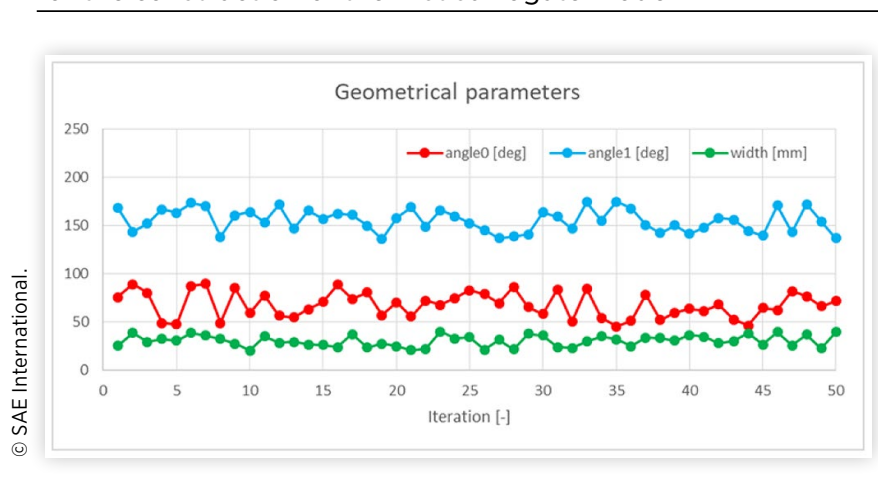

FIGURE 15 Responses of the model for each iteration, in terms of uniformity index of the ammonia distribution introduced by the mixing device.

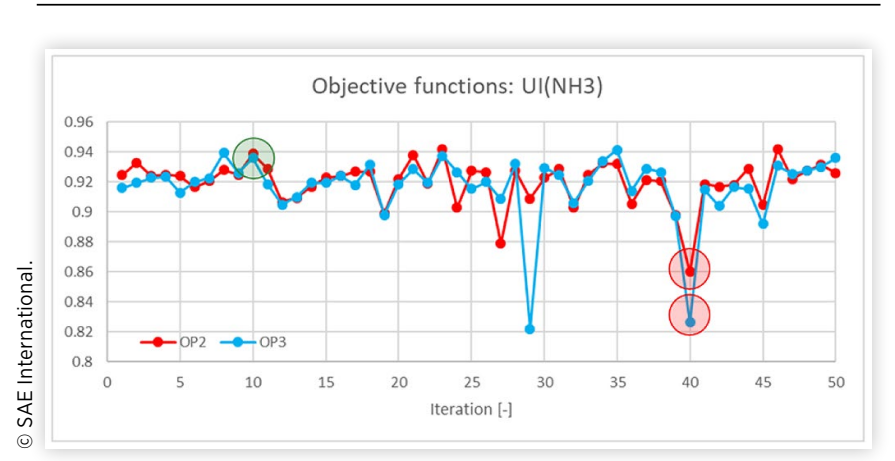


FIGURE 16 Responses of the model for each iteration, in terms of pressure drop introduced by the mixing device.

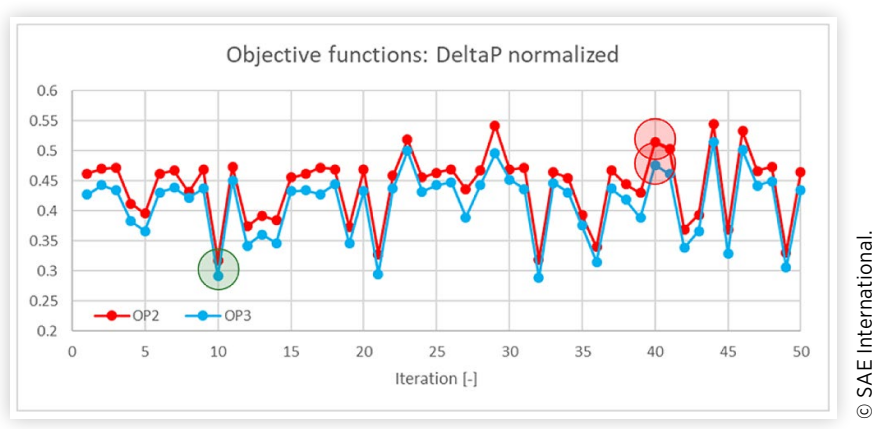

in green. On the other hand, some worst configurations can be identified, for example \#40.

The 50 points calculated in the DOE phase are exploited to build the first surrogate model consisting of additional 1000 points, as exemplified in Figure 17 - Figure 18 considering operating conditions OP2 and angle $\alpha_{0}$ and width variables. The surrogate functions allow to identify the regions in the space of the variables which are more favorable in relation to the objective of the optimization. For example, the regions highlighted in green in the graphs are characterized by low pressure drop and non-uniformity, suggesting that lower values of width and angle $\alpha_{0}$ are preferable to obtain an optimum design. On the other hand, increasing the width of the mixer and the angle $\alpha_{0}$, the pressure drop and the non-uniformity increase.

An example of optimum design is reported in Figure 19. This configuration is characterized by high uniformity of the ammonia distribution associated to a low pressure drop (included in the green region in Figure 17-Figure 18). This is obtained reducing the width of the mixer and adopting a quite small angle $\alpha_{0}$ and a high angle $\alpha_{1}$. On the other hand, in Figure 20, it can be seen that increasing the width of the mixer not only the pressure drop increases, as one would expect, but also the mixing performances are penalized.

FIGURE 17 Surrogate model built for operating condition OP2, reporting the normalized pressure drop of the mixing section as a function of the angle $\alpha_{0}$ and width variables.

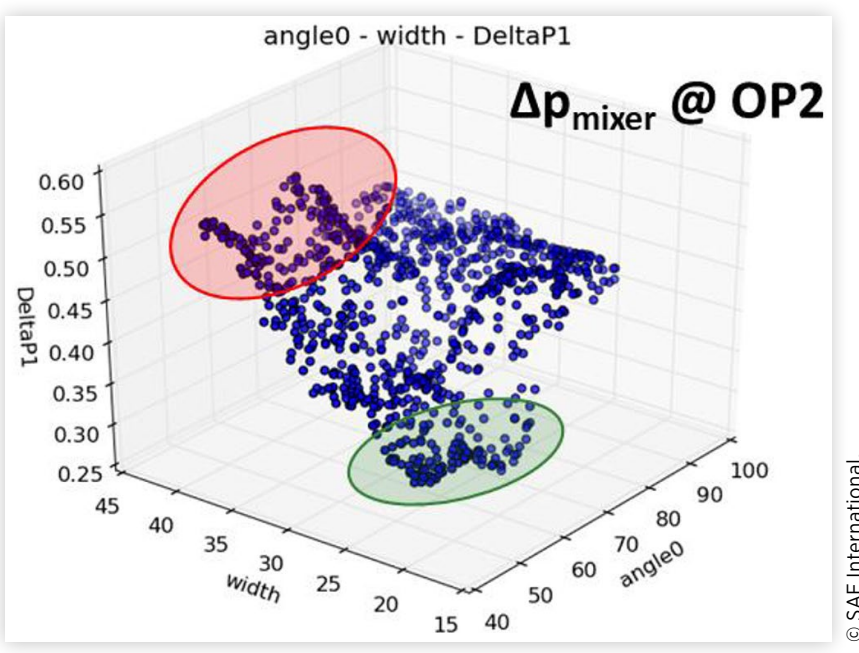

FIGURE 18 Surrogate model built for operating condition OP2, reporting the coefficient of variance of the ammonia distribution on the inlet section of SDPF as a function of the angle $\alpha_{0}$ and width variables.

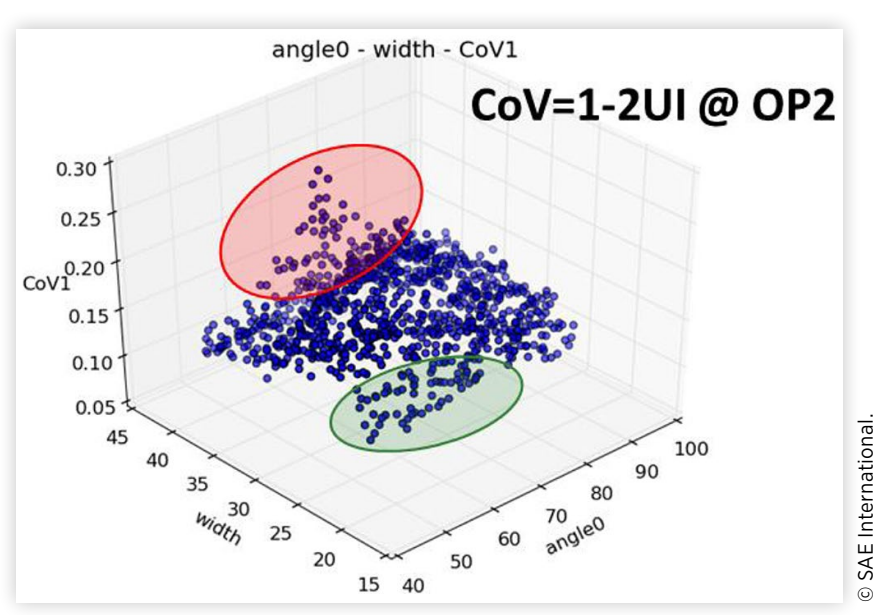

FIGURE 19 Optimal configuration: high uniformity index on the inlet section of the SDPF is obtained with low pressure drop, reducing the width of the mixer.

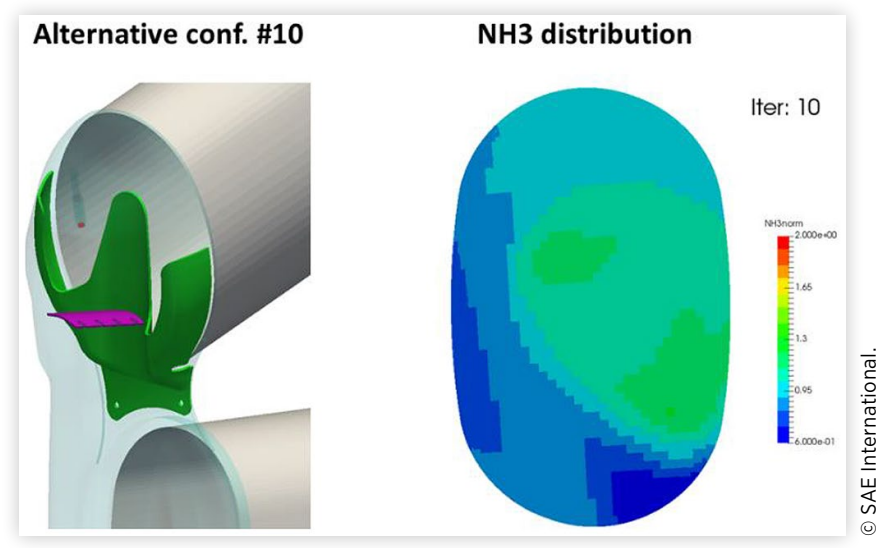

FIGURE 20 Worst configuration: this design is not efficient in promoting the mixing and it is associated to high pressure drop.

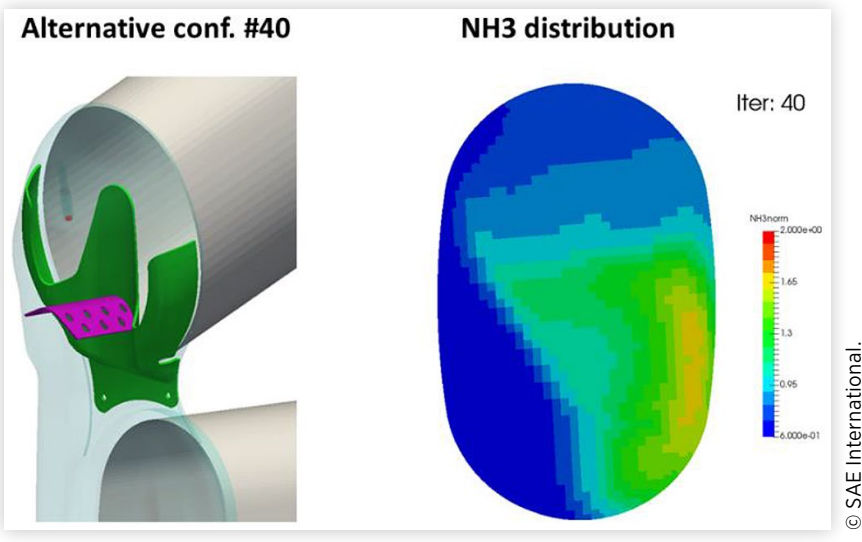


FIGURE 21 Comparison between baseline configuration and alternative configurations \#10 and \#40, in terms of uniformity of the $\mathrm{NH}_{3}$ distribution at the inlet section of the SDPF catalyst.

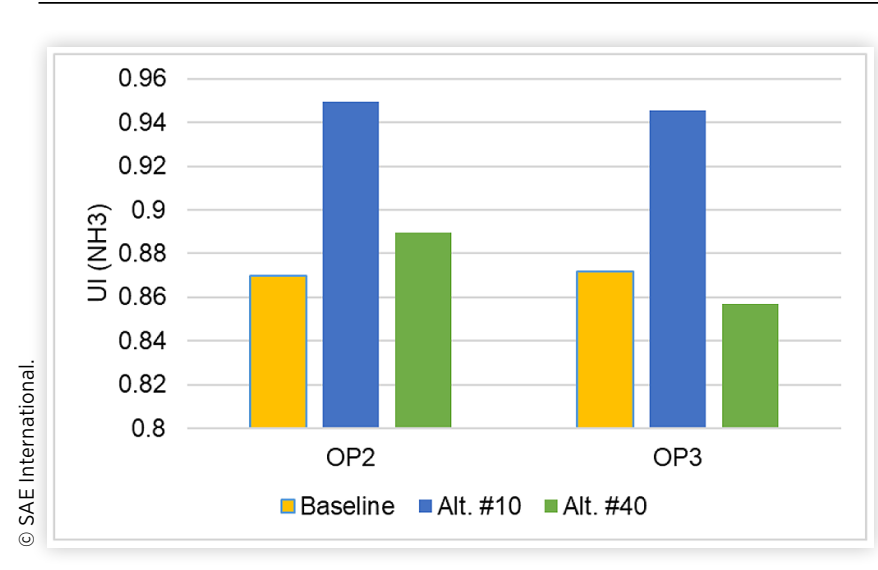

FIGURE 22 Comparison between baseline configuration and alternative configurations \#10 and \#40, in terms of pressure drop related to the mixing section.

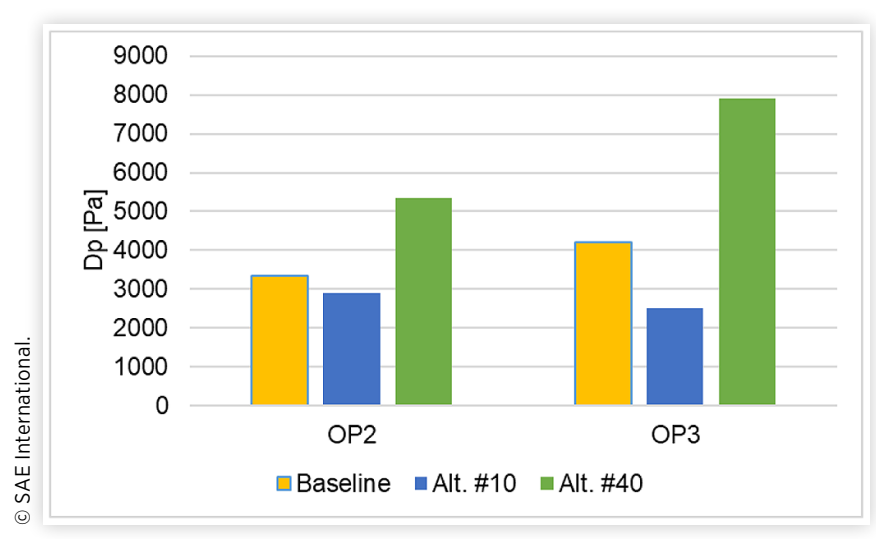

Figure 21 - Figure 22 report the detailed results in term of uniformity of the ammonia distribution and pressure drop calculated for two alternative configurations (\#10 - optimized and \#40 - not optimized) considering two operating conditions (OP2 and OP3, as detailed in Table 2). These are compared with the results calculated for the baseline configuration. It can be noticed that the alternative configuration, if properly optimized as for configuration $\# 10$, is characterized by better overall performances if compared to the baseline. As a matter of the fact it is able to obtain a higher uniformity index with lower pressure drop for both the operating conditions tested. On the other hand, if not optimized, as in the case of configuration \#40, the uniformity index is still comparable to that of the baseline configuration, while the pressure drop is significantly higher.

\section{D Analysis of the System}

The optimization process described in the previous section was focused on the stand-alone device, in order to improve its performances in terms of $\mathrm{NH}_{3}$ mixing and reduction of the pressure drop. However, in order to achieve the target of an overall optimization of the system, it is important to evaluate how the single device interacts with the other components. This target can be achieved considering a $1 \mathrm{D}$ simulation model extended to the entire exhaust line. In this framework, the components which exhibit a particular tridimensional behavior (e.g. the SDPF which is affected by a particular $\mathrm{NH}_{3}$ inlet maldistribution) can be properly modeled with the support of the information coming by detailed CFD simulation.

Simulation Model The 1D simulation model of the aftertreatment system was created using axisuite, including all the components already detailed in the previous section "Case study" as shown in Figure 23.

The model was adopted to simulate a WLTC cycle, for which experimental measurement of raw emissions from the engine were available. Two different approaches were applied for the modeling of the NH3 injection section and SDPF component. The first approach was to assume that a perfect mixing was reached at the inlet of the SDPF (i.e. $\mathrm{NH}_{3}$ uniformity index equal to 1): in this conditions the SPDF can be modeled resorting to a simple $1 \mathrm{D}$ approximation. The second approach was to take into account the $\mathrm{NH}_{3}$ maldistribution, which can be imposed considering the $2 \mathrm{D} \mathrm{NH}_{3}$ distribution maps coming from 3D simulation (e.g. Figure 5 - Figure 7) for different mass flows. In this case, the SPDF catalyst is modeled with a 3D macro-scale approach, where the computational cells are representative of a group of channels with different locations in the space. The instantaneous ammonia concentration at each location of the catalyst inlet section is determined on the basis of a specific procedure, which takes as input a set of ammonia distributions predicted by CFD simulation at different mass flows. CFD computed ammonia distributions are normalized to obtain weight factors at each location of the catalyst inlet section. In order to obtain the actual distribution for a certain mass flow rate, the model computes the actual weight factors by applying an interpolation of the weight factors available for the input mass flow rates. Once the weight factors have been determined, the actual ammonia concentration at each location is imposed on the basis of the total ammonia mass flow which should be injected depending on the prescribed dosing. Figure 24 shows the ammonia concentration at the inlet of the SDPF computed by the model for different instantaneous conditions during the WLTC cycle, with different average mass flow and NOx concentrations.

FIGURE 23 1D simulation model of the after-treatment system.

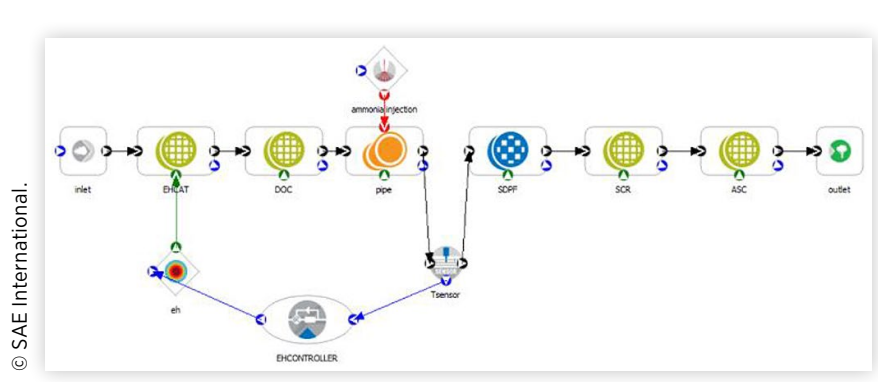


FIGURE 24 Ammonia concentration computed at different timesteps during the WLTC cycle.

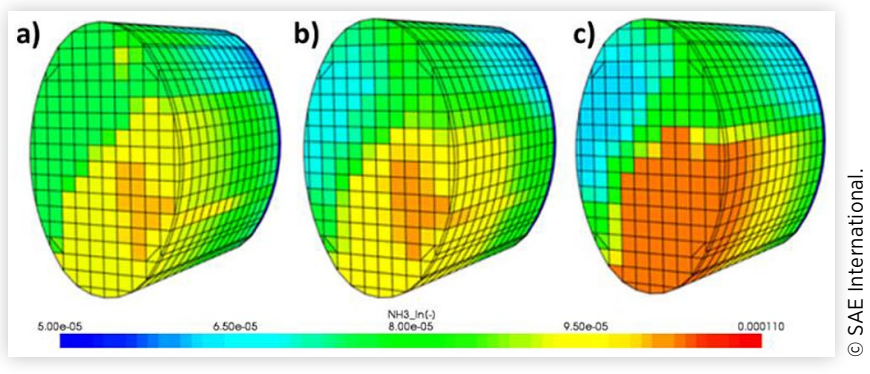

Preliminary Validation Preliminary validation was addressed considering a configuration with electrical heating switched off. For this first comparison a simple 1D approach was adopted for the modeling of the SDPF component, therefore the effect of the $\mathrm{NH}_{3}$ maldistribution was neglected. Comparison between simulation results and measurement of tailpipe NOx emission is reported in Figure 25.

It can be seen that the overall trend is well reproduced by the computational model, despite a slight overestimation of the prediction of the total NOx emission. This is related to an overestimation occurring in the first $50 \mathrm{~s}$, when the experimental data show an abatement of the NOx which, conversely, is not predicted by the model. However, apart from this initial mismatch, the conversion efficiency is correctly captured over the cycle. Therefore, the model can be considered sufficiently accurate for the purpose of the analysis, which is the investigation of the effect of the NH3 maldistribution on the overall NOx abatement efficiency. Moreover, the fact that a quite accurate prediction can be obtained neglecting the maldistribution effects can prefigure that these have a secondary importance on the overall phenomena, as will be discussed in the next section.

Effect of the $\mathrm{NH}_{3}$ Maldistribution at the SDPF Inlet In order to evaluate the impact of an alternative design of the mixing section, capable to reduce the $\mathrm{NH}_{3}$ maldistribution at the inlet of the SDPF, the 1D simulation was run adopting the 3D approach for the modeling of the SDPF. In this case, the information provided by the detailed CFD model

\section{FIGURE 25 NOx emission at the tailpipe on the WLTC} cycle: comparison between calculation and measurements. Data are non-dimensionalized with respect to a reference value.

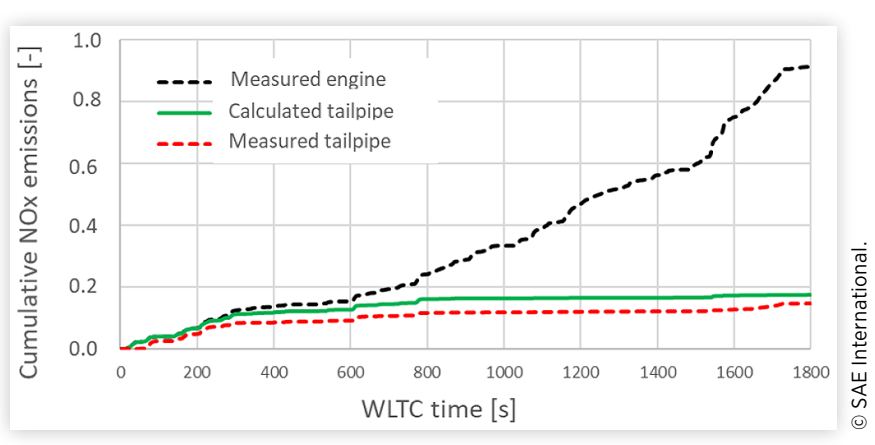

of the mixing section were exploited to provide a map for the non-uniform distribution of the NH3 at the SDPF for different operating conditions. As first step, the baseline configuration was considered, which is characterized by a uniformity index in the range $85 \%-95 \%$. The analysis was repeated for different ratio between injected $\mathrm{NH}_{3}$ and $\mathrm{NO}_{\mathrm{x}}$ content in the exhaust gas, in the range $0.8-1.5$. Figure 26 reports the decay of the NOx conversion passing from a uniform ammonia distribution to a realistic non-uniform distribution imposed on the basis of the CFD results. It can be seen that the maximum reduction of the NOx conversion is below the $2.5 \%$ after the SDPF and below $1.5 \%$ at the tailpipe. Moreover, the conversion reduction decreases with the increase of the ammonia dosing.

The fact that the effect of the $\mathrm{NH}_{3}$ maldistribution is quite limited (around $1 \%$ at the tailpipe for stoichiometric dosing) is due to the storage of ammonia on the catalyst washcoat, which amount continuously varies during the test cycle. The presence of ammonia stored on the washcoat allows to partially compensate the eventual instantaneous lean ammonia feeding of some parts of the SDPF. The storage on SDPF is possible, even in stoichiometric conditions, because this catalyst receives an excess of $\mathrm{NH}_{3}$ compared to the maximum conversion which is achieved on average during the cycle, taking into account that the full reduction process is completed in the second SCR underfloor. Ammonia storage varies during the test cycle and increases when the activity of the catalyst is low, for example at the cold start, while it decreases during the high speed part of the cycle, remaining however always present.

Moreover, the second factor which compensates the maldistribution is related to the presence of the second SCR in underfloor position, which receives the ammonia well mixed with the exhaust gas flow and therefore contributes to the further reduction of the residual $\mathrm{NO}_{\mathrm{x}}$.

This analysis suggests that the effort towards the increase of the mixing efficiency could be not justified in this configuration, since the presence of the second SCR stage allows to mitigate in a quite effective way the effects of the eventual maldistribution. On the other hand, it points out that the optimization of the device should be addressed considering not only the stand-alone component but also taking into account its interaction with the system.

FIGURE 26 DeNOx performance after SDPF and the tailpipe, for different ratio $\mathrm{NH}_{3} / \mathrm{NO}_{x}$ : decay of the performance passing from uniform ammonia distribution (ID SDPF model) to the realistic distribution predicted by CFD model for baseline mixer configuration.

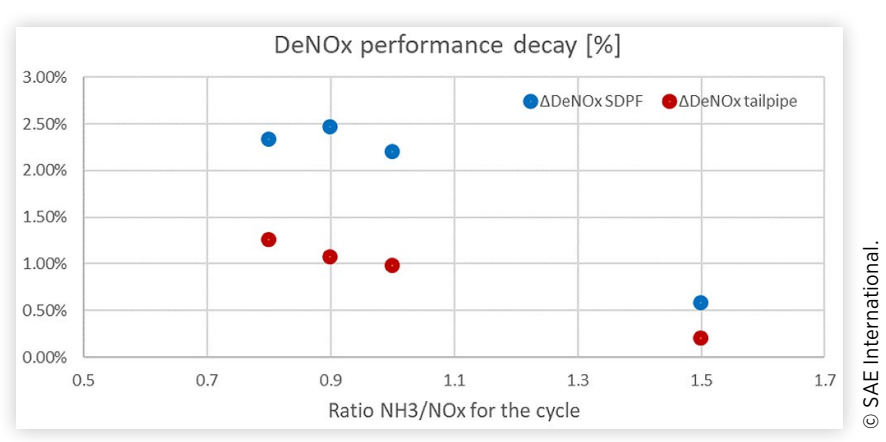




\section{Conclusions}

In this paper, the modeling of an after-treatment system for a Diesel light-duty engine has been addressed. The study has been conducted with a suitable integration of $1 \mathrm{D}$ and CFD numerical tools, in order to characterize the system at different level of details. In particular, a detailed CFD model was applied to simulate the behavior of the mixer, which is crucial for promoting the mixing between ammonia and exhaust gas and, therefore, the overall DeNOx performance of the system. The role of the CFD model was, primarily, to provide the information to be exploited in the $1 \mathrm{D}$ code to improve the model and to embed a more detailed characterization taking into account the effective distribution of the ammonia at the SDPF inlet. Moreover, the CFD model was adopted to perform the optimization of the mixing device in order to obtain a more uniform ammonia distribution. To this purpose, an optimization framework was setup, suitably coupling OpenFOAM with Dakota, in order to modify some key geometrical parameters of the mixer in such a way to minimize the objective functions, namely pressure drop and $\mathrm{NH}_{3}$ maldistribution. This procedure led to a new optimized design, characterized by significantly better performances with respect to the baseline. However, the analysis of the impact of this improvement on the overall system DeNOx performance showed a negligible contribution. In this framework, the 1D analysis was fundamental to clarify the reasons of this unexpected behavior, which is related to the presence of two NOx reduction stages and to the effects of the ammonia storage on the SDPF. Therefore, this case study showed the importance of adopting an integrated approach to guide the optimization, in order to exchange information at the different level of detail (component and system) in such a way to evaluate the impact of the component behavior on the overall system performances.

\section{References}

1. Johnson, T., "Diesel Emissions in Review," SAE Int. J. Engines 4(1):143-157, 2011.

2. Tsinoglou, D. and Koltsakis, G., "Modelling of the Selective Catalytic NOx Reduction in Diesel Exhaust Including Ammonia Storage," Proceedings of the Institution of Mechanical Engineers, Part D: Journal of Automobile Engineering 221(1):117-133, 2007.

3. Tsinoglou, D., Koltsakis, G., and Jones, G.P., "Oxygen Storage Modeling in Three-Way Catalytic Converters," Industrial \& Engineering Chemistry Research 41(5):1152-1165, 2002.

4. Cerri, T., D'Errico, G., Montenegro, G., Onorati, A. et al., "A Novel 1D Co-Simulation Framework for the Prediction of Tailpipe Emissions under Different IC Engine Operating
Conditions," SAE Technical Paper 2019-24-0147, 2019, https://doi.org/10.4271/2019-24-0147.

5. Della Torre, A., Montenegro, G., Onorati, A., and Cerri, T., "CFD Investigation of the Impact of Electrical Heating on the Light-Off of a Diesel Oxidation Catalyst," SAE Technical Paper 2018-01-0961, 2018, https://doi.org/10.4271/2018-01-0961.

6. Nova, I. and Tronconi, E., Urea-SCR Technology for deNOx After Treatment of Diesel Exhausts (New York: Springer, 2014).

7. OpenFOAM, “OpenFOAM Documentation," http://www. openfoam.org/docs/.

8. Weller, H., Tabor, G., Jasak e C., H., and Fureby, "A Tensorial Approach to CFD Using Object Orientated Techniques," Computers in Physics 12(6):620, 1998.

9. Dakota, "Dakota Documentation," in https://dakota.sandia. gov/documentation.html.

10. Koltsakis, G., Konstantinidis, P., and Stamatelos, A., "Development and Application Range of Mathematical Models for 3-Way Catalytic Converters," Applied Catalysis B: Environmental 212(2-3):161-191, 1997.

11. Versteeg, H. and Malalasekera, W., An Introduction to Computational Fluid Dynamics (Longman Scientific \& Technical, 1995).

12. Menter, F.R., "Two-Equation Eddy-Viscosity Turbulence Models for Engineering Applications," AIAA Journal 32(8):1598-1605, 1994.

\section{Contact Information}

\author{
Dr. Augusto Della Torre \\ Department of Energy, Politecnico di Milano \\ Via Lambruschini, 4 \\ 20156 Milano, Italy \\ phone: +390223998631 \\ augusto.dellatorre@polimi.it
}

\section{Acknowledgments}

The authors gratefully acknowledge financial support from the Horizon 2020 EU Research and Innovation Programme, within the project THOMSON: Mild hybrid cost effective solutions for a fast market penetration (Grant Agreement no. 724037).

\section{Definitions/Abbreviations}

\author{
eHC - Electrical heated catalyst \\ DOC - Diesel Oxidation Catalyst \\ SDPF - SCR on DPF \\ SCR - Selective Catalytic Reduction \\ ASC - Ammonia Slip Catalyst
}

(c) 2020 SAE International. All rights reserved. No part of this publication may be reproduced, stored in a retrieval system, or transmitted, in any form or by any means, electronic, mechanical, photocopying, recording, or otherwise, without the prior written permission of SAE International. 